\title{
Analysis of Fatigue Behavior of High-Density Polyethylene Based on Nonlinear Viscoelastic Measurement under Cyclic Fatigue
}

\author{
Nam-Ju Jo, Atsushi Takahara, and Tisato KajiYama* \\ Department of Chemical Science \& Technology, Faculty of Engineering, \\ Kyushu University, Higashi-ku, Fukuoka 812, Japan
}

(Received December 21, 1992)

\begin{abstract}
Fatigue behaviors of annealed and isothermally crystallized high-density polyethylenes (HDPEs) were investigated on the basis of nonlinear dynamic viscoelastic measurement during the fatigue process. Nonlinear viscoelasticity was evaluated in terms of nonlinear viscoelastic parameter (NVP) which was defined as the contribution of higher harmonics of Fourier expanded stress signal. In the cases of the annealed and isothermally crystallized HDPEs, the apparent dynamic storage modulus decreased, NVP and $\tan \delta$ increased with an increase in the magnitude of imposed strain amplitude. Since the fatigue strength decreased when the contribution of NVP became dominant, the magnitude of NVP can be used as a measure of fatigue characteristics of polymeric solids. Also, the origin of NVP for the annealed and isothermally crystallized HDPEs was discussed on the basis of the spherulite structure.

KEY WORDS Fatigue Behavior / High-Density Polyethylene / Nonlinear Viscoelasticity / Dynamic Viscoelasticity / Nonlinear Viscoelastic Parameter / Crystalline State /
\end{abstract}

In the last decades, various polymers have been used for structural components as the high-strength and high modulus materials. Thus, it is very important to clarify the fatigue behavior of such polymeric materials in order to investigate long term reliability for engineering applications. Also, under the conditions which ultimately lead to fatigue failure, nonlinear viscoelastic behavior becomes prominent, because polymeric materials are subjected to a large imposed strain amplitude which is larger than that of the linear viscoelastic limit. Therefore, it seems reasonable to consider that fatigue failure mechanism is related to the nonlinear viscoelasticity.

Prevorsek and co-workers investigated the nonlinear viscoelasticity of nylon fibers in terms of the changes in modulus, internal friction, and structure. ${ }^{1,2}$ Onogi and Matsumoto

\footnotetext{
* To whom correspondence should be addressed.
}

measured the nonlinear viscoelastic properties of dispersion systems in solutions and evaluated nonlinear viscoelastic parameters from resultant torque. ${ }^{3,4}$ Also, Onogi and Tanaka studied the nonlinear viscoelastic behavior of solid polymeric materials on the basis of the simultaneous measurements of dynamic viscoelasticity and dynamic birefringence under large cyclic deformation. ${ }^{5}$ They supposed that the nonlinear viscoelasticity of HDPE at a low temperature was caused by both the orientation of amorphous region and slippage of crystallites, and also, at a high temperature, by the orientation and slippage of crystallites. The nonlinear stress relaxation behavior in simple extension for crosslinked polyester resins was investigated by Tieghi and co-workers. ${ }^{6}$ And also, So and Chen have studied creep and stress relaxation of solid- 
filled rubbers, and proposed a nonlinear mechanical model consisting of four nonlinear elements $^{7}$. It has been reported that the nonlinear viscoelasticity is related to the irreversible structural change induced by a large imposed strain. However, little study has been done on the relationship between fatigue behaviors and nonlinear viscoelasticity. The authors proposed fatigue fracture criterion based on hysteresis energy loss for solid polymers under cyclic straining. ${ }^{8,9}$ This hysteresis energy loss was divided into two parts such as the energy dissipated as a heat generation and that used for a structural change. It has been observed that the fraction of energy loss dissipated for a structural change in total hysteresis energy loss increased with the magnitude of imposed strain amplitude. The average hysteresis energy loss dissipated for a structural change, $H_{\text {s,av }}$ during cyclic fatigue was evaluated, and then, the empirical relationship between $H_{\mathrm{s} \text {,av }}$ and fatigue lifetime, $t_{\mathrm{f}}$ represented by eq 1 was established as the fatigue criterion.

$$
\left(H_{\mathrm{s}, \mathrm{av}}-H_{0}\right) t_{f}=C
$$

where $H_{0}$ is the fatigue limit hysteresis energy loss and $C$ is the total hysteresis loss up to fatigue failure. However, this study has only provided the information about the apparent and gross nonlinear viscoelasticity.

In this study, the evaluation method of nonlinear viscoelasticity for high-density polyethylene (HDPE) during the fatigue process was proposed on the basis of Fourier analysis of stress signal, and the relationship between fatigue behavior and nonlinear viscoelasticity was also discussed.

\section{EXPERIMENTAL}

\section{Specimens}

The polymer used in this study was highdensity polyethylene (HDPE, Hizex 1300J, Mitsui Petrochemical Co., Ltd.) with a melt index of 14.0. HDPE was compression molded into molten-films with about $0.5 \mathrm{~mm}$ in thickness at $453 \mathrm{~K}$ and then, the molten-films were quenched by plunging them into ice water. The 'annealed' sample was obtained by annealing the quenched film at $393 \mathrm{~K}$ for $5 \mathrm{~h}$ under nitrogen atmosphere. The 'isothermally crystallized' sample was prepared by the isothermal crystallization at $388 \mathrm{~K}$ for $5 \mathrm{~h}$. The degree of weight fraction crystallinity, $\chi_{\mathrm{c}}$ estimated by density measurements for the annealed and isothermally crystallized samples was 0.72 and 0.78 , respectively.

\section{Wide Angle X-Ray Diffraction}

For the purpose of analysis of crystalline state of samples, wide angle X-ray diffractograms were obtained at room temperature using a Ni-filtered $\mathrm{Cu}-K_{\alpha}$ radiation $(\lambda=$ $0.1542 \mathrm{~nm}$ ) which was generated from a rotating anode source $(40 \mathrm{kV}, 200 \mathrm{~mA})$. Assuming that the distribution function for lattice distortions and the shape factor are Lorentzian, the crystal size, $D_{h k l}$ and the lattice distortion, $g_{h k l}$ were evaluated on the basis of the Hosemann's paracrystal analysis. ${ }^{10}$

\section{Fatigue Testing}

Figure 1 shows the blockdiagram of fatigue tester to investigate nonlinear dynamic viscoelastic properties. The fatigue test was carried out by using the fatigue tester (VFA-20: Orientec Co., Ltd.) which enabled continuous measurement of dynamic viscoelastic functions during the fatigue process. The strain and stress signals were measured by the semiconductor displacement sensor and load cell, respectively. $E^{\prime}$ was calculated from $\left|E^{*}\right|$ which has obtained from the apparent magnitude of dynamic stress amplitude and dynamic strain amplitude. The magnitude of mechanical loss tangent, $\tan \delta$ was obtained from the phase difference between the stress and strain signals under cyclic straining, where $\delta$ was the apparent phase difference between the stress and strain signals. Here, the distortion of the stress signal was not considered. Surface temperature 


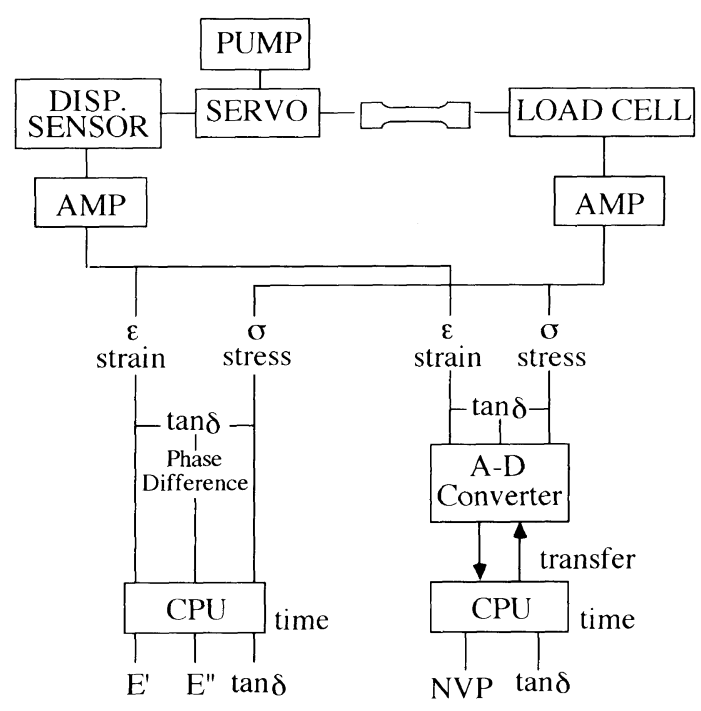

Figure 1. Blockdiagram of fatigue tester for investigation of nonlinear dynamic viscoelastic properties under cyclic fatigue.

was monitored by using infrared radiation thermometer. Fatigue tests were performed under the conditions of the testing frequency of $2 \mathrm{~Hz}$ and the ambient temperature of $303 \mathrm{~K}$. The magnitude of dynamic strain amplitude, $\varepsilon_{\mathrm{d}}$ was in the range of $0.2-1.8 \%$. The magnitude of initial static stress was varied from $10-20 \mathrm{MPa}$. In the analysis of nonlinear viscoelasticity, the imposed strain and response stress signals were collected with an A-D converter. This A-D converter has a large memory so that this can be used as a digital storage memory. The digital data were transferred to the personal computer, and nonlinear viscoelastic property was estimated based on the procedure mentioned in the following section.

\section{Estimation of Nonlinear Viscoelastic Property}

When the sinusoidal dynamic strain, $\varepsilon_{0}$ with angular frequency, $\omega=2 \pi f$ is imposed on the static strain, $\varepsilon_{s}$, the total strain, $\varepsilon(t)$ is expressed by eq 2 .

$$
\varepsilon(t)=\varepsilon_{\mathrm{s}}+\varepsilon_{0} \sin \omega t
$$

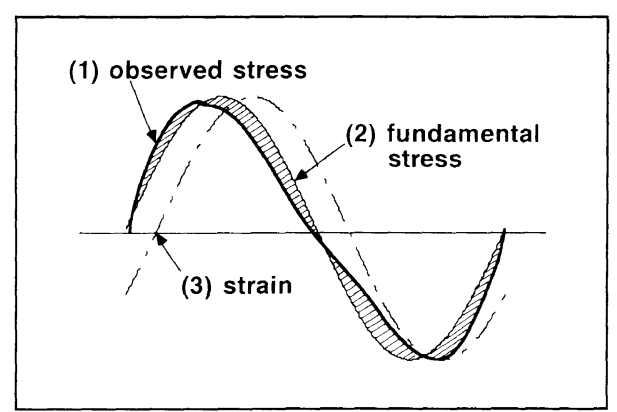

Figure 2. Schematic representation of nonlinear viscoelastic response under constant sinusoidal strain amplitude. The thick solid line, the thin solid line, and the chain line indicate observed stress wave (1), fundamental stress wave (2), and strain wave (3), respectively.

At a large imposed strain amplitude, the response stress signal would be distorted in comparison with the fundamental stress signal as shown in Figure 2. Therefore, it is apparent that the response stress, $\sigma(t)$ can be expressed by Fourier expansion as follows.

$$
\begin{aligned}
\sigma(t)= & a_{0} / 2 \\
& +a_{1} \cos \omega t+a_{2} \cos 2 \omega t+a_{3} \cos 3 \omega t \cdots \\
& +b_{1} \sin \omega t+b_{2} \sin 2 \omega t+b_{3} \sin 3 \omega t+\cdots
\end{aligned}
$$

where the coefficients of Fourier series, $a_{0}, a_{n}$, and $b_{n}$ can be calculated by eq 4,5 , and 6 , respectively.

$$
\begin{gathered}
a_{0}=(\omega / \pi) \int_{0}^{2 \pi / \omega} \sigma(t) \mathrm{d} t \\
a_{n}=(\omega / \pi) \int_{0}^{2 \pi / \omega} \sigma(t) \cos (n \omega t) \mathrm{d} t \\
b_{n}=(\omega / \pi) \int_{0}^{2 \pi / \omega} \sigma(t) \sin (n \omega t) \mathrm{d} t \\
(n=1,2, \cdots)
\end{gathered}
$$

In this experiment, the magnitudes of $a_{0}, a_{n}$, and $b_{n}$ were evaluated from the numerical integral of a cyclic stress signal. The fundamental stress wave (wave(2) in Figure 2) was composed of the fundamental components $\left(\sigma(t)=a_{1} \cos \omega t+b_{1} \sin \omega t\right)$. It has been reported 
that the deviation of the observed stress wave (wave (1) in Figure 2) from the fundamental stress wave apparently corresponded to the degree of nonlinear viscoelasticity. ${ }^{5}$ Therefore, the degree of nonlinear viscoelastic property can be represented by the nonlinear viscoelastic parameter, NVP being defined as follows:

$N V P=$

$$
\frac{\sqrt{a_{2}^{2}+b_{2}^{2}}+\sqrt{a_{3}^{2}+b_{3}^{2}}+\cdots+\sqrt{a_{10}^{2}+b_{10}^{2}}}{\sqrt{a_{1}^{2}+b_{1}^{2}}}
$$

Since the values of the 11 th order coefficient or above were very small, then the contributions from these terms were neglected.

\section{RESULTS AND DISCUSSION}

\section{Crystalline State of the Annealed and Isotherm- ally Crystallized HDPE}

Table I shows the crystal structural parameters evaluated from the paracrystal theory ${ }^{10}$ for as-pressed, annealed, and isothermally crystallized HDPEs. The coherently diffracting lengths (crystallite sizes), $D_{110}$ and $D_{200}$ in the 110 and 200 directions of the annealed HDPE were somewhat smaller than those of the isothermally crystallized HDPE. The lattice distortion factors, $g_{110}$ and $g_{200}$ in the 110 and 200 directions of the annealed HDPE were larger than those of the isothermally crystallized one. Therefore, it is apparent from Table I that the crystallite of the annealed HDPE is slightly distorted compared with that of the isothermally crystallized HDPE. A polarizing optical microscopic observation of the annealed and the isothermally crystallized HDPEs revealed that the isothermally crystallized HDPE has larger spherulites and sharper spherulite boundary than the annealed one. Creep of the annealed HDPE during the fatigue process was greater than that of the isothermally crystallized HDPE. Also, the authors observed from the small angle light scattering that the spherulite of the HDPE with indistinct spherulite interface was deformed, while that
Table I. Crystal structural parameters of as-pressed, annealed, and isothermally crystallized HDPEs

\begin{tabular}{|c|c|c|c|c|}
\hline \multirow{2}{*}{ Sample } & $D_{110}$ & $D_{200}$ & $g_{110}$ & $g_{200}$ \\
\hline & $\mathrm{nm}$ & $\mathrm{nm}$ & $\%$ & $\%$ \\
\hline As-pressed HDPE & 27.7 & 20.2 & 1.15 & 1.29 \\
\hline Annealed HDPE & 29.2 & 23.8 & 1.16 & 1.20 \\
\hline $\begin{array}{l}\text { Isothermally } \\
\text { crystallized HDPE }\end{array}$ & 32.7 & 30.1 & 0.72 & 1.15 \\
\hline
\end{tabular}

\section{Annealed HDPE}

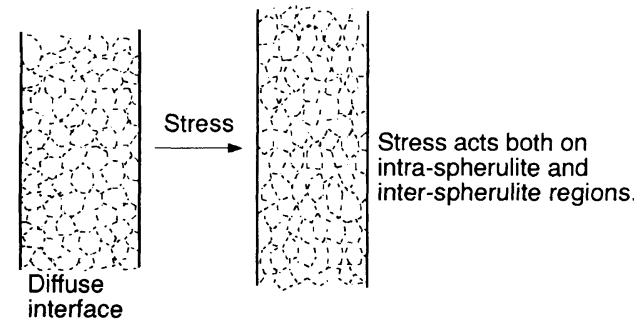

Isothermally Crystallized HDPE

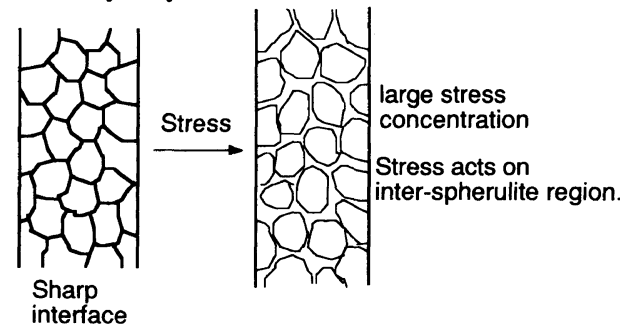

Figure 3. Schematic representation of spherulite state and stress concentration region for the annealed and isothermally crystallized HDPE.

of the HDPE with distinct spherulite interface was not deformed under the cyclic fatigue. ${ }^{11}$ Figure 3 shows a schematic representation of the initial crystalline states obtained on the basis of microscopic observations and the deformation mechanism under the cyclic fatigue for the annealed and isothermally crystallized HDPEs. In the case of the annealed HDPE, the spherulite interface was not sharp, and its spherulite size was smaller compared with that of the isothermally crystallized HDPE, resulting in a fairly amount of interfacial connection among spherulites. Therefore, an imposed 
dynamic strain acts on both intra-spherulite and inter-spherulite, and leads to spherulite deformation. On the other hand, in the case of the isothermally crystallized HDPE, an imposed dynamic strain concentrated on the spherulite interface, because a mechanically week interfacial region forms a distinct boundary. Therefore, fatigue fracture was preferentially proceeded at the spherulite interface region. The deformation studies mentioned above indicate that it is interesting to study the effect of cyclic fatigue on nonlinear viscoelasticity for the annealed and isothermally crystallized HDPEs.

\section{Nonlinear Viscoelastic Behavior of Annealed HDPE during the Fatigue Process}

Figure 4 shows the variations of the dynamic storage modulus, $E^{\prime}$, the mechanical loss tangent, $\tan \delta$, and the nonlinear viscoelastic parameter, NVP for the annealed HDPE with time during the fatigue process at the initial static stress of $9.8 \mathrm{MPa}$ as a function of magnitude of imposed dynamic strain amplitudes. The magnitudes of $E^{\prime}$ and $\tan \delta$ were apparent ones which included the contribution of higher order term of response stress. Thus, NVP which reflects the contribution of higher order term has been introduced. A surface temperature rise was slight owing to the large surface to volume ratio of the specimen. Then, the changes in $E^{\prime}, \tan \delta$, and NVP under cyclic fatigue may be attributed mainly to the structural origin. The magnitude of $E^{\prime}$ decreased and that of $\tan \delta$ increased with an increase in imposed strain amplitude. The increase in $\tan \delta$ with imposed strain amplitude might be due to the contribution of nonlinear viscoelasticity, because an increase in viscous contribution to mechanical loss factor may be neglected due to almost constant specimen temperature during cyclic fatigue. In the case of smaller imposed strain amplitude, $E^{\prime}$ showed maximum and $\tan \delta$ did minimum just before the fatigue failure, though these tendencies were not apparent in Figure 4. These are typical

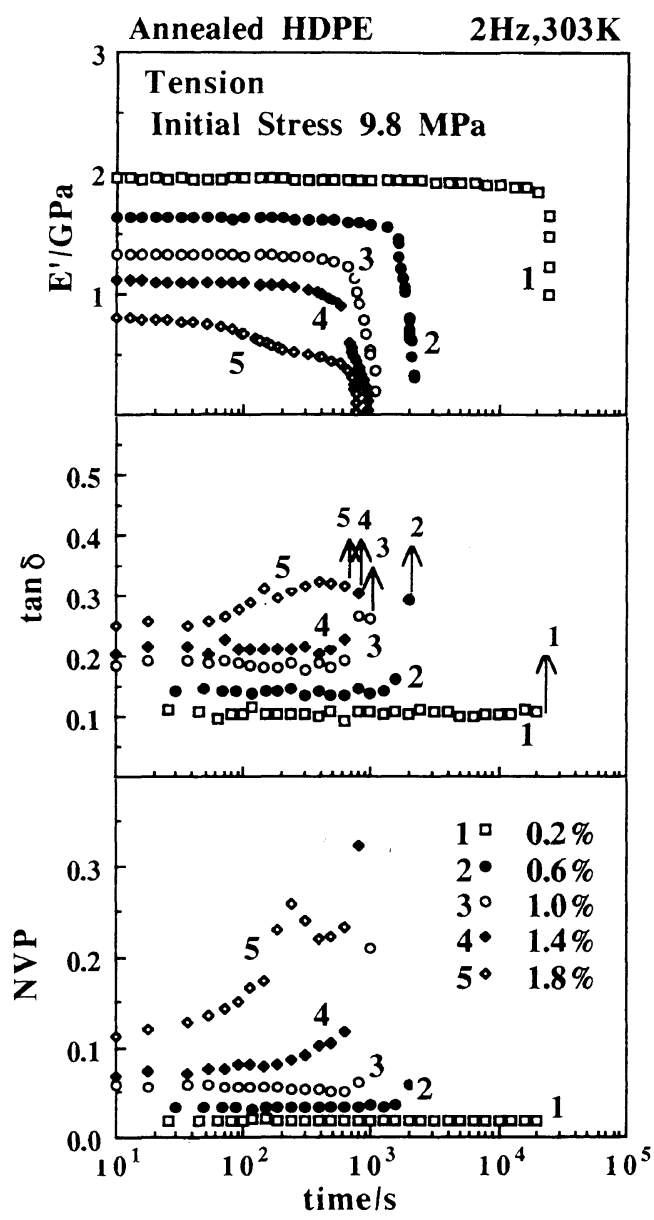

Figure 4 Variations of $E^{\prime}, \tan \delta$, and NVP for the annealed HDPE with time during the fatigue process at the initial static stress of $9.8 \mathrm{MPa}$ under various dynamic imposed strain amplitudes.

behaviors for brittle failure, and indicate considerable increase in contribution of elastic term, such as orientation of molecular chains under cyclic fatigue. ${ }^{12,13}$ At larger imposed strain amplitude, $E^{\prime}$ decreased and $\tan \delta$ increased monotonously from the start of fatigue process. This is a typical behavior of ductile failure. ${ }^{12,13}$ The magnitude of NVP increased consistently with that of imposed strain amplitude. Therefore, it may be concluded that nonlinear viscoelastic behavior became prominent at larger imposed strain amplitude, accompanying a decrease in $E^{\prime}$ and 


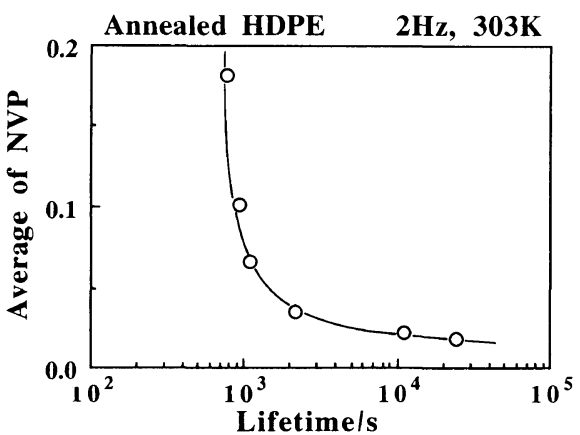

Figure 5 Relationship between fatigue lifetime and the average of NVP for the annealed HDPE.

an increase in $\tan \delta$.

Figure 5 shows the relationship between the fatigue lifetime and the average of NVP from the onset of cyclic fatigue until the fatigue failure for the annealed HDPE. The average of NVP increased with imposed dynamic strain amplitude and the fatigue lifetime remarkably decreased with an increase in NVP. Also, since the fatigue strength of the annealed HDPE decreases when the nonlinear viscoelasticity becomes more prominent, the average magnitude of NVP can be applicable for a measure of fatigue characteristics of polymeric solids.

Figure 6 provides the variations of $E^{\prime}, \tan \delta$, and NVP for the annealed HDPE with time during the fatigue process at the dynamic imposed strain amplitude of $1.0 \%$ under various magnitudes of static stress. It was observed that $E^{\prime}$ slightly decreased and $\tan \delta$ slightly increased with an increase in initial static stress. On the other hand, the variation of NVP was almost independent of the change of initial static stress, except for a final stage of fatigue failure. That is, nonlinear behavior became prominent on approaching the point of failure. Simultaneous measurements of dynamic viscoelasticity and small angle light scattering pattern during the fatigue process revealed that a large increase in creep occurred just before fatigue failure accompanying deformation of spherulite. ${ }^{11}$ Thus, these results indicate that the increment of NVP is

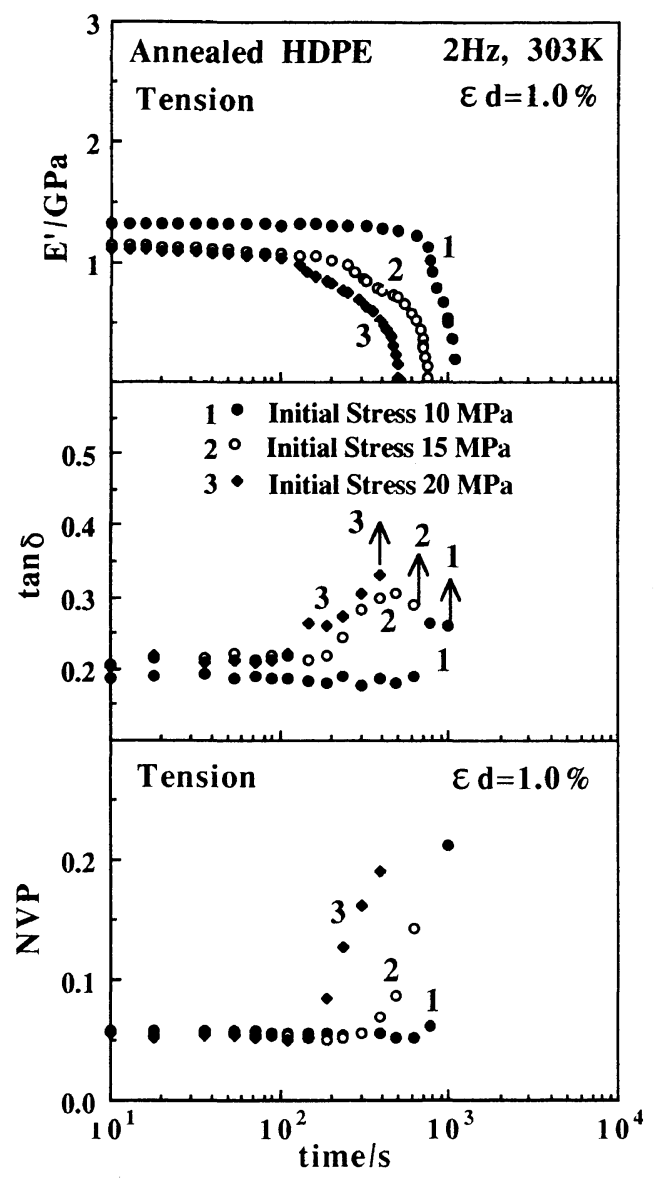

Figure 6. Variations of $E^{\prime}$, tan $\delta$, and NVP for the annealed HDPE with time during the fatigue process at the imposed dynamic strain amplitude of $1.0 \%$ under various initial static stresses.

caused by structural changes such as the orientation of amorphous region and/or the spherulite deformation.

\section{Nonlinear Viscoelasticity of Isothermally Crys- tallized HDPE during the Fatigue Process}

The isothermally crystallized HDPE was used to investigate the effect of weight fraction crystallinity and sharpness of spherulite boundary on nonlinear viscoelasticity under cyclic fatigue. Figure 7 shows the variations of $E^{\prime}, \tan \delta$, and NVP for the isothermally crystallized HDPE with time during the fatigue process at the initial static stress of $9.8 \mathrm{MPa}$ 


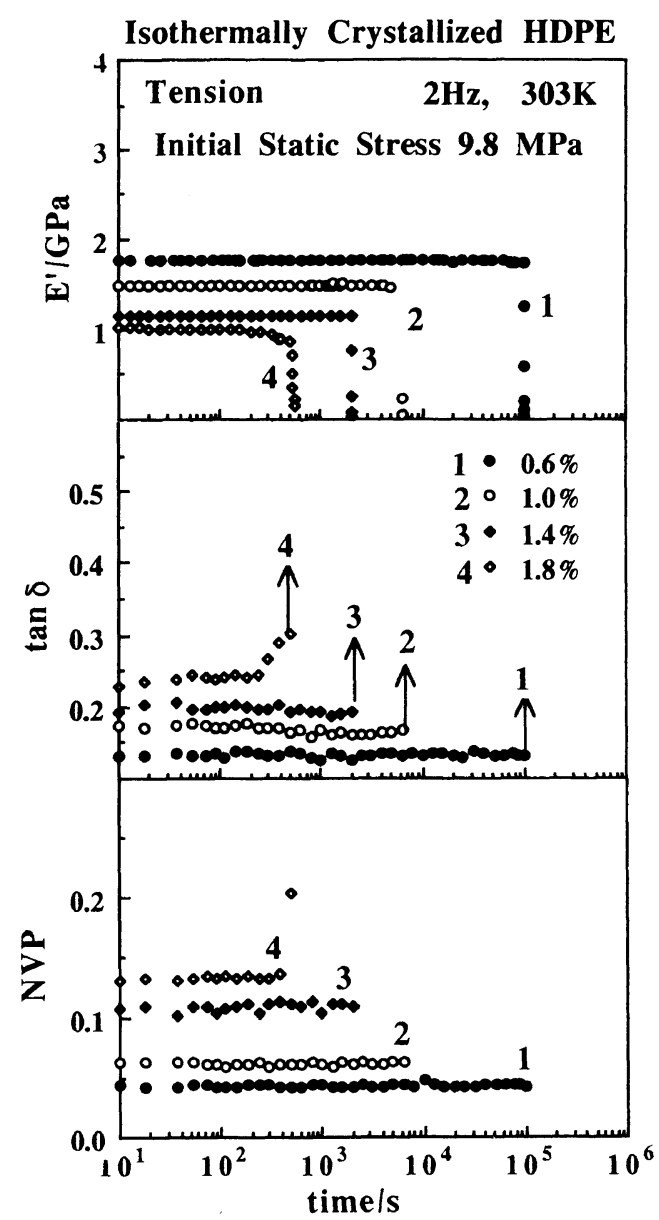

Figure 7. Variations of $E^{\prime}, \tan \delta$, and NVP for the isothermally crystallized HDPE with time during the fatigue process at the initial static stress of $9.8 \mathrm{MPa}$ under various dynamic imposed strain amplitudes.

under various imposed dynamic strain amplitudes. The magnitudes of $E^{\prime}$ and $\tan \delta$ decreased and increased, respectively, with an increase in dynamic imposed strain amplitude. This result is in a similar fashion to those of the annealed HDPE. Also, the nonlinear viscoelastic parameter, NVP increased consistently with the magnitude of imposed dynamic strain amplitude. This indicates that the fatigue behavior is strongly dependent on the nonlinear viscoelastic characteristics. The fatigue failure of the isothermally crystallized HDPE occurred abruptly at the final stage of

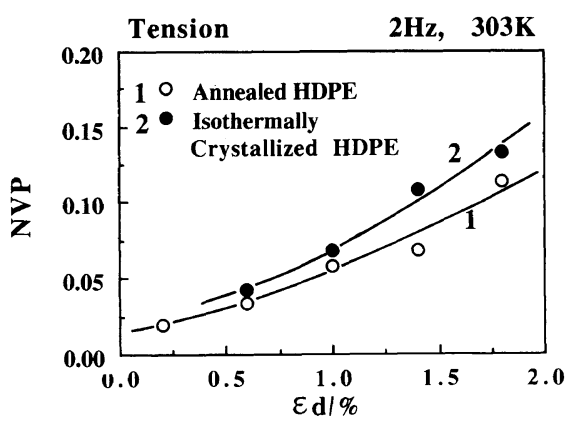

Figure 8. Relationship between the imposed dynamic strain amplitude and the magnitude of NVP after $10 \mathrm{~s}$ from the starting of fatigue test for the annealed and isothermally crystallized HDPE.

fatigue process, while that of the annealed HDPE occurred accompanying a gradual decrease in $E^{\prime}$ as shown in Figure 4. Since the spherulite boundary or crystallite interfaces in the isothermally crystallized HDPE were sharper compared with that of annealed one as schematically shown in Figure 3, these boundary or interfaces act as stress concentration regions, resulting in appearance of abrupt fracture.

Figure 8 shows the relationship between the magnitude of NVP after $10 \mathrm{sec}$ from the starting of fatigue test and the magnitude of imposed strain amplitude for the annealed and isothermally crystallized HDPEs. The magnitude of NVP for the isothermally crystallized HDPE was greater than that for the annealed one for the same magnitude of imposed strain amplitude. Since the isothermally crystallized HDPE has distinct spherulites or crystallite boundary compared with that for the annealed HDPE, the larger magnitude of NVP can be ascribed to the greater stress concentration at the spherulite boundary which induces prominently an irreversible structural change.

Figure 9 shows the relationship between fatigue lifetime and the average of NVP from the start of cyclic fatigue until the fatigue failure for the isothermally crystallized HDPE. Fatigue lifetime decreased with an increase in NVP, that was almost a similar tendency to 


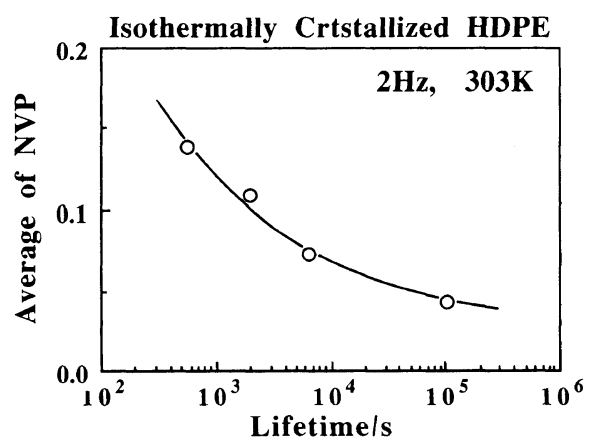

Figure 9. Relationship between fatigue lifetime and the average of NVP for the isothermally crystallized HDPE.

the result of the annealed HDPE. The fatigue strength of the isothermally crystallized HDPE decreased with increasing the nonlinear viscoelasticity same as the result of the annealed HDPE. Therefore, it was evident that the average magnitude of NVP could be used as a measure of fatigue characteristics of polymeric material.

Figure 10 shows the time dependence of $E^{\prime}$, $\tan \delta$, and NVP for the isothermally crystallized HDPE under cyclic fatigue at the imposed dynamic strain amplitude of $1.0 \%$ as a function of different initial static stresses. An increment of initial static stress did not make the magnitudes of $E^{\prime}, \tan \delta$, and NVP changed remarkably, especially in the initial stage of fatigue test. Also, the magnitude of creep during cyclic fatigue showed only a slight increase with an increase in the initial static stress. The fatigue fracture of the isothermally crystallized HDPE abruptly proceeded, accompanying the relatively fast crack propagation, while that of the annealed one occurred accompanying the spherulite deformation, as indicated in Figure 6 by a gradual decrease in $E^{\prime}$ in a final step of fatigue fracture. These differences can be reasonably expected from an apparent increase in creep under cyclic fatigue, as follows. The magnitude of initial static stress did not clearly affect the nonlinear viscoelastic property for the isothermally crystallized HDPE, because an intra-spherulite

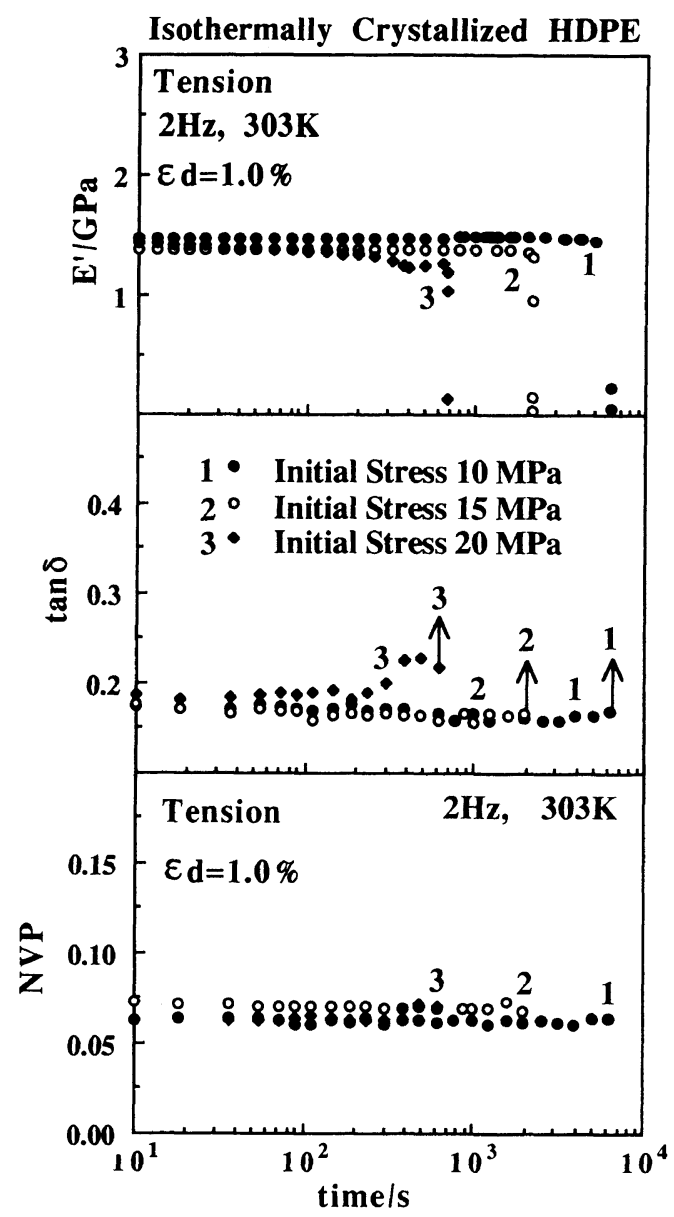

Figure 10. Variations of $E^{\prime}, \tan \delta$, and NVP for the isothermally crystallized HDPE with time during the fatigue process at the imposed dynamic strain amplitude of $1.0 \%$ under various initial static stresses.

region (spherulite itself) was not preferentially deformed due to the stress concentration at the spherulite interface. This deformation process might not lead to an increase in NVP. However, since an increase in the initial static stress more accelerated the rate of the crack propagation proceeding at the spherulite interface, the fatigue lifetime decreased with an increase in the initial static stress.

\section{CONCLUSIONS}

The effect of nonlinear viscoelasticity on 
fatigue behavior of HDPE was studied on the basis of the nonlinear viscoelastic parameter (NVP) which was evaluated from the magnitude of higher harmonics of Fourier expanded stress signal. Since a large decrease in fatigue strength with an increase in NVP has been observed for both annealed and isothermally crystallized HDPEs, the magnitude of NVP can be applicable for a measure of fatigue strength of polymeric solids. In the case of the annealed HDPE, as the imposed dynamic strain acted on both intra-spherulite and inter-spherulite (interface) regions, the magnitude of NVP was strongly dependent on spherulite deformation. On the other hand, in the case of the isothermally crystallized HDPE with sharp spherulite boundary, the magnitude of NVP might be fairly related to the degree of stress or strain concentration at the spherulite or crystallite interface which induces an irreversible structural change.

\section{REFERENCES}

1. D. C. Prevorsek, Y. D. Kwon, and R. K. Sharma, J. Macromol. Sci. -Phys., B13, 571 (1977).

2. D. C. Prevorsek, Y. D. Kwon, and R. K. Sharma, J. Appl. Polym. Sci., 25, 2063 (1980).

3. S. Onogi, T. Masuda, and T. Matsumoto, Trans. Soc. Rheol., 14, 275 (1970).

4. T. Matsumoto, Y. Segawa, Y. Warashina, and S. Onogi, Trans. Soc. Rheol., 17, 47 (1973).

5. A. Tanaka, M. Fukuda, H. Nagai, M. Shinohara, and S. Onogi, J. Polym. Sci., B, Polym. Phys., 27, 2283 (1989).

6. G. Tieghi, M. Levi, A. Fallini, and F. Danusso, Polymer, 32, 39 (1991).

7. H. So and U. D. Chen, Polym. Eng. Sci., 31, 410 (1991).

8. N. Kaiya, M. Kugishima, A. Takahara, and T. Kajiyama, Sen-i Gakkaishi, 42, T-127 (1986).

9. N. Kaiya, A. Takahara, and T. Kajiyama, Polym. J., 21, 523 (1989).

10. R. Hosemann and W. Wilke, Makromol. Chem., 118, 230 (1968).

11. A. Takahara, Y. Nishi, N. Kaiya, and T. Kajiyama, J. Soc. Rheol. Jpn., 19, 72 (1991).

12. A. Takahara, K. Yamada, T. Kajiyama, and M. Takayanagi, J. Appl. Polym. Sci., 25, 597 (1980).

13. A. Takahara, K. Yamada, T. Kajiyama, and M. Takayanagi, J. Appl. Polym. Sci., 26, 1085 (1981). 